\title{
On calculating disconnected-type hadronic light-by-light scattering diagrams from lattice QCD
}

\section{Hayakawa*}

Department of Physics, Nagoya University, Japan

Nishina Center, RIKEN, Japan

\section{T. Blum}

Physics Department, Connecticut University, USA

RIKEN-BNL Research Center, Brookhaven National Laboratory, USA

\section{N. H. Christ}

Physics Department, Columbia University, USA

\section{T. Izubuchi}

Physics Department, Brookhaven National Laboratory, USA

RIKEN-BNL Research Center, Brookhaven National Laboratory, USA

\section{C. Jin}

Physics Department, Columbia University, USA

\section{Lehner}

Physics Department, Brookhaven National Laboratory, USA

For reliable comparison of the standard model prediction to the muon $g-2$ with its experimental value, the hadronic light-by-light scattering (HLbL) contribution must be calculated by lattice QCD simulation. HLbL contribution has many types of disconnected-type diagrams. Here, we start with recalling the point that must be taken care of in every method to calculate them by lattice QCD, and present one concrete method called nonperturbative QED method.

The 33rd International Symposium on Lattice Field Theory

14 - 18 July 2015

Kobe International Conference Center, Kobe, Japan

\footnotetext{
*Speaker.
} 


\section{Introduction}

The hadronic light-by-light scattering (HLbL) contribution, $a_{\mu}(\mathrm{HLbL})$, will leave a controversial uncertainty in the standard model prediction $a_{\mu}($ th) to the muon $g-2$, unless it can be calculated by means of lattice QCD simulation.

The reason is as follows. While the hadronic vacuum polarization (HVP) contribution to the muon $g-2$ can resort to the experiments to evaluate the relevant QCD dynamics, $a_{\mu}(\mathrm{HLbL})$ requires purely theoretical consideration. Thus far, it has been only estimated according to the models with several hadrons such as pions as dynamical variables. The value of $a_{\mu}(\mathrm{HLbL})$ in Tab. 1 was obtained as such [1]. Including it as the part of $a_{\mu}($ th), we observe the discrepancy

Table 1: Comparison of the discrepancy between theory and experiment with HLbL contribution. All are given in units of $10^{-11}$.

\begin{tabular}{rr}
\hline \hline$a_{\mu}(\exp )-a_{\mu}(\mathrm{th})$ & $249(87)$ \\
\hline$a_{\mu}(\mathrm{HLbL})$ & $116(40)$ \\
\hline$\delta a_{\mu}($ next $\exp )$ & $O(1)$ \\
\hline
\end{tabular}
between the experiment and $a_{\mu}(\mathrm{th})$, which is comparable in size with $a_{\mu}(\mathrm{HLbL})$. Actually, no proof supporting the validity of the low energy approximation to $a_{\mu}(\mathrm{HLbL})$ exists. There is thus a potential possibility of significance of QCD dynamics that cannot be captured by hadron models. Therefore, the first-principle calculation with quarks and gluon as dynamical variables, such as lattice QCD simulation, is crucial to provide $a_{\mu}(\mathrm{HLbL})$ with manageable theoretical uncertainty.

Recently, feasibility was demonstrated to compute the HLbL contribution by the lattice simulation [2], and more efficient method is investigated in Ref. [3]. Mainz group has attempted to calculate the HLbL amplitude [4]. All of those works, however, focus on so-called connected-type diagram shown in Fig. 1, where all of four electromagnetic (EM) vertices lie on a single quark loop.

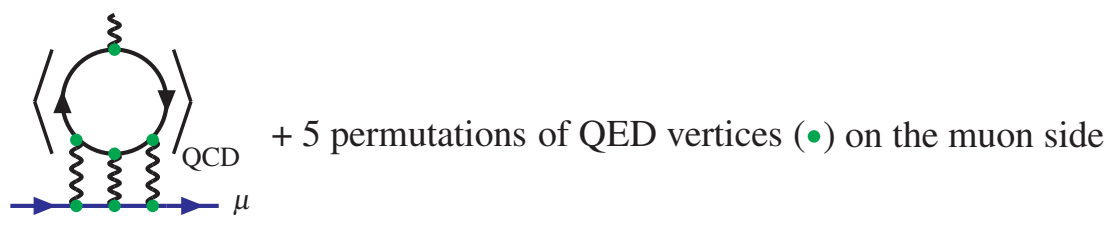

Figure 1: Connected-type HLbL diagrams. Each quark line under the QCD average represents the inverse of the quark Dirac operator $\mathrm{D}[U]$ for a given QCD configuration $U$. The diagrams with $O(a)$ local QED vertices are not shown here.

Here we turn our attention to the disconnected-type HLbL contribution whose details are presented in Sec. 2. We first see the point that must be called into account in every method to compute it by lattice simulation in Sec. 3. In Sec. 4, we also present one concrete method with such a point taken into account by remedying the one proposed in Ref. [5].

\section{Classification of disconnected-type HLbL diagrams}

The lattice QCD simulation tempts us into classifying the diagrams according to the way how four EM vertices are distributed over quark loops. It turns out that there are seven types of disconnected-type diagrams in total. The diagrams of type $\left(3_{E}, 1\right)$ in Fig. 2 are those with three EM vertices on a quark loop, one of which couples to the external photon, and one internal EM vertex on the other loop. The diagrams of type $\left(1_{E}, 3\right)$ in Fig. 3 differ from those of type $\left(3_{E}, 1\right)$ because 

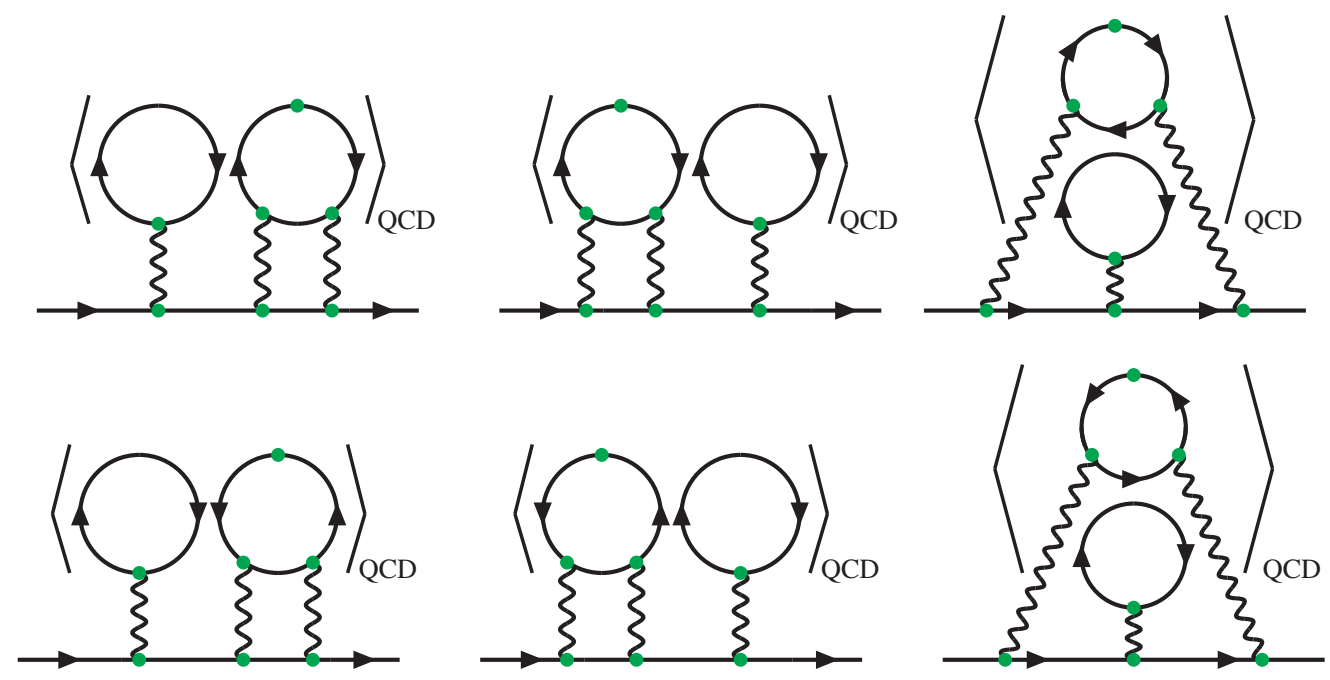

Figure 2: $\left(3_{E}, 1\right)$-type diagrams. The diagrams with $O(a)$ local QED vertices are not shown.
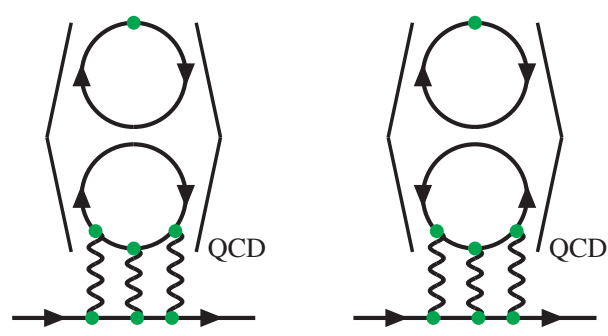

Figure 3: $\left(1_{E}, 3\right)$-type diagrams
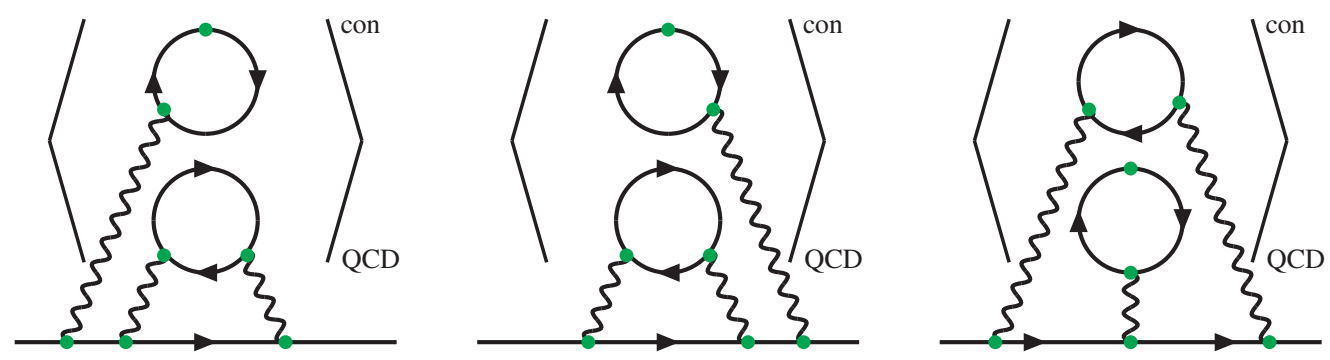

Figure 4: $\left(2_{E}, 2\right)$-type diagrams

there is no internal EM vertex on the quark loop with the external EM vertex. The diagrams of type $\left(2_{E}, 2\right)$ in Fig. $4{ }^{1}$ are also the disconnected-type diagrams having just two quark loops with two internal EM vertices on each. The diagrams in Figs. 5 and 6 are those having three quark loops with at least one EM vertex on each. The diagram in Figs. 7 has four quark loops with just one EM vertex on each.

\footnotetext{
${ }^{1}$ The meaning of the superscript "con" attached to the average symbol will be clarified in Sec. 3 .
} 


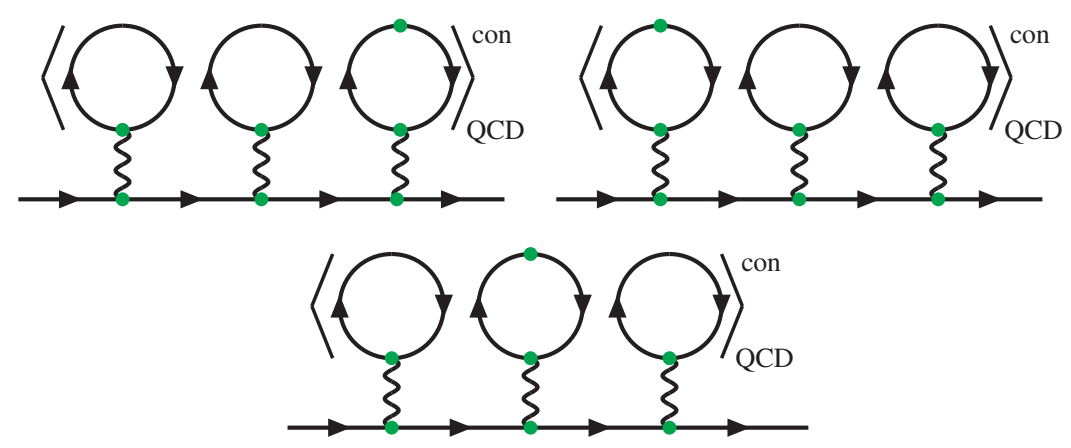

Figure 5: $\left(2_{E}, 1,1\right)$-type diagrams
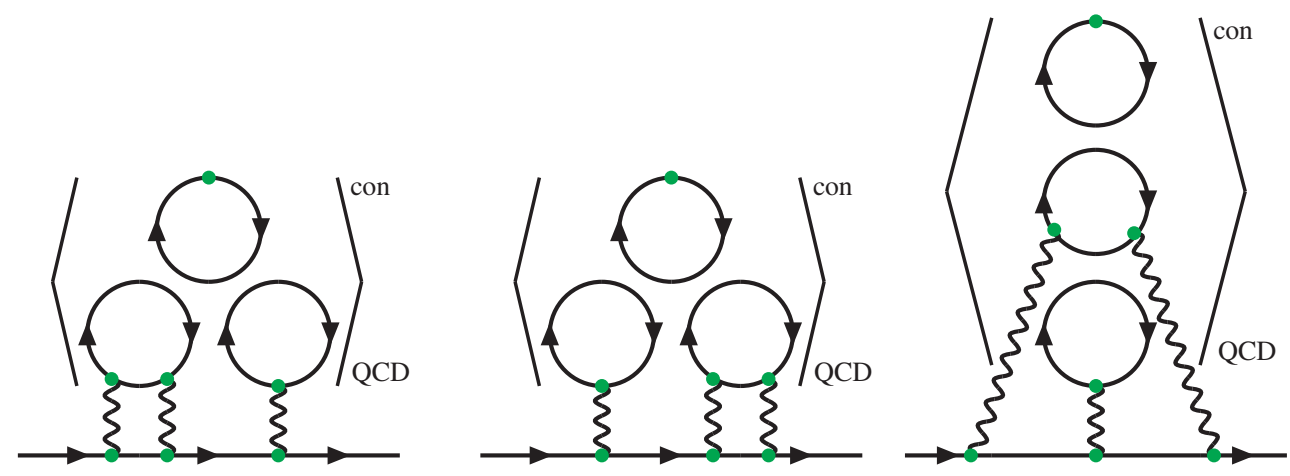

Figure 6: $\left(1_{E}, 1,2\right)$-type diagrams

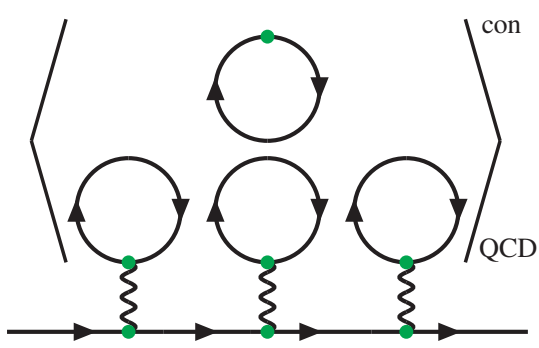

Figure 7: $\left(1_{E}, 1,1,1\right)$-type diagrams

\section{Disconnected component in the correlation function of four EM currents}

Lattice QCD simulation may enable to compute the vacuum expectation value (VEV) of four hadronic EM currents $j_{\mu}(x)$

$$
\begin{aligned}
& \left\langle j_{\mu_{(1)}}\left(x_{(1)}\right) j_{\mu_{(2)}}\left(x_{(2)}\right) j_{\mu_{(3)}}\left(x_{(3)}\right) j_{\mu_{(4)}}\left(x_{(4)}\right)\right\rangle_{\mathrm{QCD}} \\
& \quad=\frac{1}{Z_{\mathrm{QCD}}} \int d U \int d q d \bar{q} e^{-S_{\mathrm{QCD}}[U, q, \bar{q}]} j_{\mu_{(1)}}\left(x_{(1)}\right) j_{\mu_{(2)}}\left(x_{(2)}\right) j_{\mu_{(3)}}\left(x_{(3)}\right) j_{\mu_{(4)}}\left(x_{(4)}\right) .
\end{aligned}
$$

This VEV, however, contains not only the contribution from the field-theoretically connected diagrams, but also that from the field-theoretically disconnected diagrams. Here, a field-theoretically 
disconnected diagram is a Feynman diagram consisting of more than one nontrivial connected subgraph as a graph. We shall refer to the contribution of field-theoretically disconnected (connected) diagrams as the disconnected (connected) component of that contribution. If the connected component of the QCD average of $\mathscr{A}$ is denoted by $\langle\mathscr{A}\rangle_{\mathrm{QCD}}^{\mathrm{con}}$, the quantity relevant to the HLbL is $\left\langle j_{\mu_{(1)}}\left(x_{(1)}\right) j_{\mu_{(2)}}\left(x_{(2)}\right) j_{\mu_{(3)}}\left(x_{(3)}\right) j_{\mu_{(4)}}\left(x_{(4)}\right)\right\rangle_{\text {QCD }}^{\text {con }}$, which differs from the one in Eq. (3.1) by the sum of three terms each of which is a product of two currents, $\left\langle j_{\lambda}(x) j_{\rho}(y)\right\rangle_{\mathrm{QCD}}$.

Basically, lattice QCD simulation does not allow us to evaluate $\langle\mathscr{A}\rangle_{\mathrm{QCD}}^{\mathrm{con}}$ directly because connectivity is the attribute of each Feynman diagram with quarks and gluons. The best we can do is to compute $\langle\mathscr{A}\rangle_{\mathrm{QCD}}$ and its disconnected component $\langle\mathscr{A}\rangle_{\mathrm{QCD}}^{\text {dis }}$ to get $\langle\mathscr{A}\rangle_{\mathrm{QCD}}^{\mathrm{con}}=\langle\mathscr{A}\rangle_{\mathrm{QCD}}-\langle\mathscr{A}\rangle_{\mathrm{QCD}}^{\text {dis }}$ indirectly.

If the VEV of four EM currents in Eq. (3.1) couples to the muon via three virtual photons, its disconnected component gives rise to the HVP contribution with the $O(\alpha)$ renormalization of EM charge due to QCD. Any calculation of the HVP contribution with the renormalized EM charge contains such a contribution implicitly. To avoid double counting, we must thus explicitly subtract such an $O\left(\alpha^{3}\right)$ HVP contribution, which is henceforth called unwanted contribution, in every method based on lattice QCD. Subtraction is required in practice to compute the disconnected-type diagrams; $\left(2_{E}, 2\right),\left(1_{E}, 1,2\right),\left(2_{E}, 1,1\right)$ and $\left(1_{E}, 1,1,1\right)$ in our classification scheme.

\section{Nonperturbative QED method for full HLbL contribution}

The nonperturbative QED method we propose here to compute full HLbL contribution is given by

$$
\frac{1}{3}\left\{\left(\mathscr{M}_{C}-\mathscr{S}_{C}\right)+\left(\mathscr{M}_{C^{\prime}}-\mathscr{S}_{C^{\prime}}\right)+\left(\mathscr{M}_{D}-\mathscr{S}_{D}\right)-\mathscr{K}_{D}\right\}
$$

where the terms $\mathscr{M}_{C}, \mathscr{S}_{C}, \mathscr{M}_{C^{\prime}}$ and $\mathscr{S}_{C^{\prime}}$ are defined in Fig. 8, and $\mathscr{M}_{D}, \mathscr{S}_{D}$ and $\mathscr{K}_{D}$ in Fig. 9. The individual terms involve the averages with respect to (QCD + QED) for light quark system. Note that each muon line in Figs. 8 and 9 denotes the inverse of muon Dirac operator in the QED configuration generated by such $(\mathrm{QCD}+\mathrm{QED})$ system. We multiply $\frac{1}{3}$ to the quantity in the bracket in Eq. (4.1) because individual 8 types

Table 2: Emergence of degeneracy. $C$, say, denotes $\mathscr{M}_{C}-\mathscr{S}_{C}$.

\begin{tabular}{l|c|c}
\hline \hline & $C+C^{\prime}$ & $D$ \\
\hline $4_{E}$ & 3 & 0 \\
$\left(1_{E}, 3\right)$ & 0 & 3 \\
$\left(3_{E}, 1\right)$ & 2 & 1 \\
$\left(2_{E}, 2\right)$ & 1 & 2 \\
$\left(1_{E}, 1,2\right)$ & 0 & 3 \\
$\left(2_{E}, 1,1\right)$ & 1 & 2 \\
$\left(1_{E}, 1,1,1\right)$ & 0 & 3 \\
\hline
\end{tabular}
of HLbL diagrams emerge with triplicate degeneracy as in Tab. 2 [5].

The term $\left(-\mathscr{K}_{D}\right)$ in Eq. (4.1) is the one added here to subtract the unwanted $O\left(\alpha^{3}\right)$ HVP contribution contained in the other terms. To construct $\mathscr{K}_{D}$, we prepare two sets of (QCD, QED). Practically, they may be two independent important samples of a pair of $(U, A)$ generated by dynamical (QCD + QED) simulation. The quark in the upper loop on the right-hand side of Fig. 9 is charged only with respect to the first (QCD, QED) and the one in the lower loop only with respect to the second (QCD, QED), while the muon is charged with respect to both QEDs.

We discuss how subtraction of unwanted contributions is realized by $\left(-\mathscr{K}_{D}\right)$. For that purpose, it may be helpful to observe the situation by focusing on a $\left(2_{E}, 2\right)$-type diagram. It is generated in three ways as shown in Fig. 10 ; each diagram in Fig. 10 contains the unwanted contribution, i.e. $O\left(\alpha^{3}\right)$ HVP contribution. Note that the HVP contribution coming from $\mathscr{M}_{C}$ can be canceled 

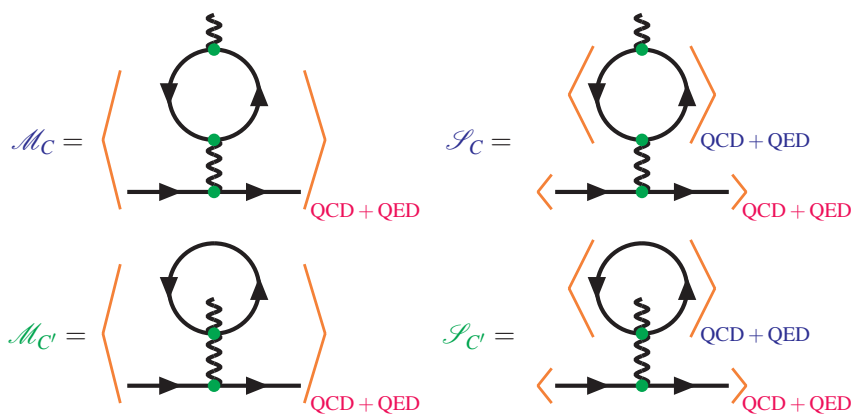

Figure 8: The terms $\mathscr{M}_{C}, \mathscr{S}_{C}$, and $\mathscr{M}_{C^{\prime}}, \mathscr{S}_{C^{\prime}}$ with $O(a)$ QED vertices.

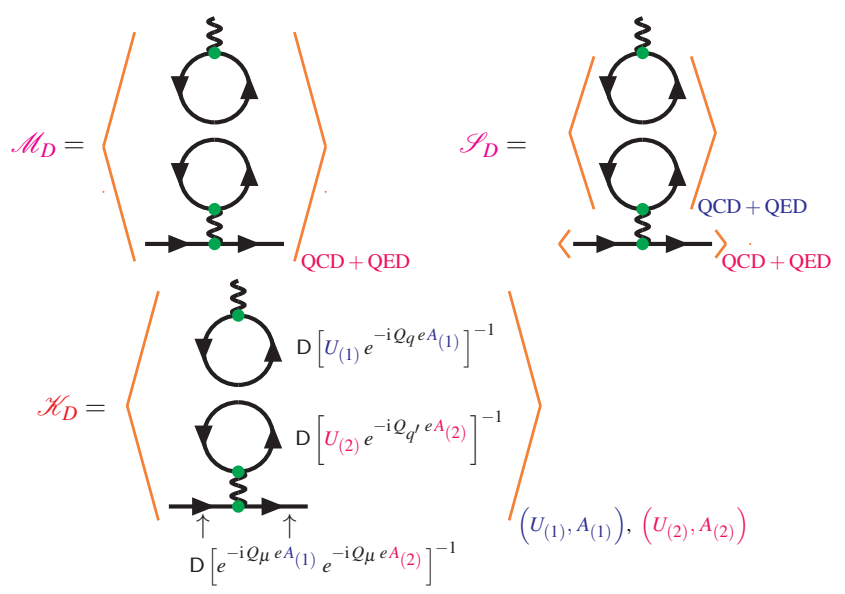

Figure 9: The terms $\mathscr{M}_{D}, \mathscr{S}_{D}$ and $\mathscr{K}_{D}$.
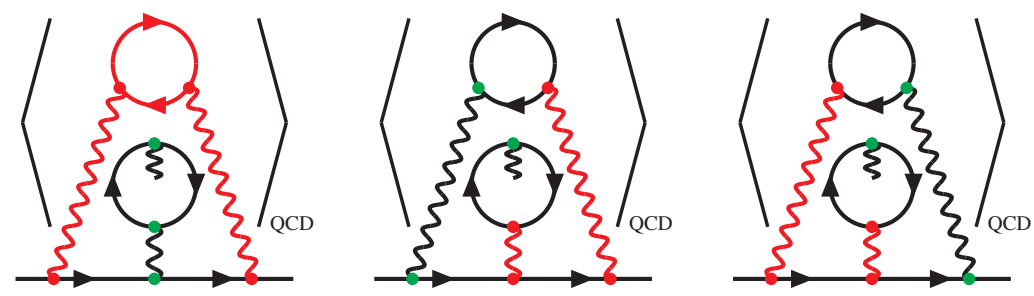

Figure 10: An identical diagram of $\left(2_{E}, 2\right)$-type is generated in three ways from $\mathscr{M}_{C}$ (left) and $\mathscr{M}_{D}$ (middle, right). The red propagators and vertices are generated by the ensemble average of (QCD + QED) .

by that supplied from $\mathscr{S}_{C}$, but the other two survive. The essential difference between them is as follows. The HVP contribution canceled by $\mathscr{S}_{C}, \mathscr{S}_{C^{\prime}}$ or $\mathscr{S}_{D}$ contains one HVP function entirely supplied from QED average (QCD average of fully red quark loop), but the uncanceled one does not. The same is true for the other disconnected-type diagrams. If the full HVP function is denoted as in Fig. 11, the unwanted contributions that survive in the absence of the last term in Eq. (4.1) can be summarized in Fig. 12, where each diagram of identical topology turns out to appear exactly twice. One can show that a set of the $O\left(\alpha^{3}\right)$-diagrams generated by $\mathscr{K}_{D}$ exactly coincides with that in Fig. 12 with the same degeneracy, verifying that subtraction is realized in the nonperturbative QED method. 


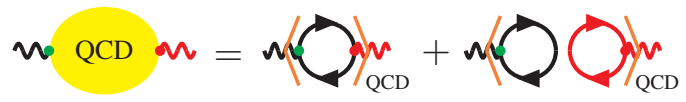

Figure 11: Full HVP function. The diagrams with $O(a)$ QED vertices are not shown.
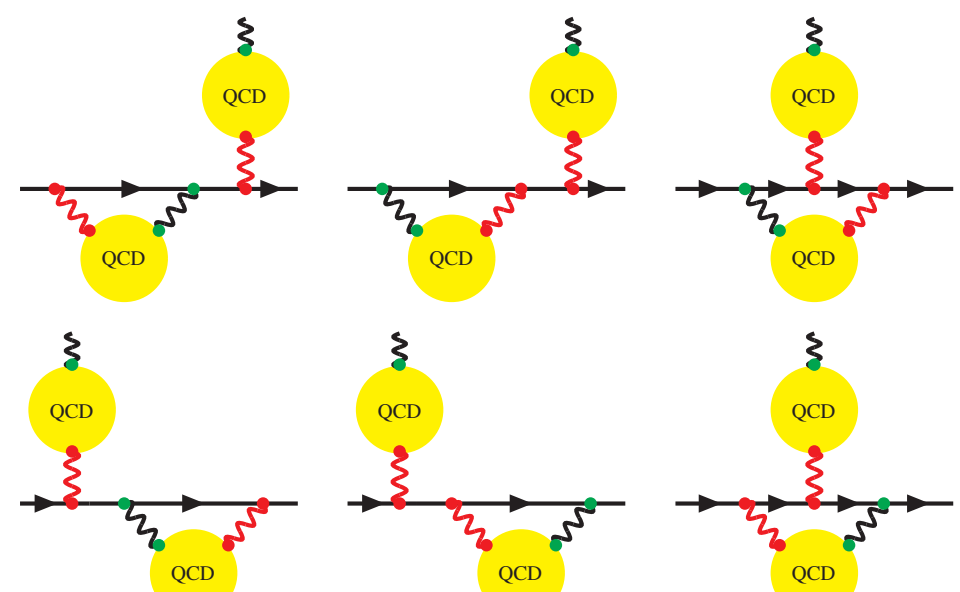

Figure 12: Summary of $O\left(\alpha^{3}\right)$ unwanted diagrams.

\section{Summary}

Here, we remarked that, to avoid double counting in the prediction of the muon $g-2$, we must explicitly subtract $O\left(\alpha^{3}\right)$ HVP contributions in every method for the computation of full HLbL contribution. We presented an idea (4.1) for the concrete method, which is based on the dynamical (QCD + QED) simulation as done in Ref. [6], though the results in Ref. [3] encourage to develop alternative methods without stochastic realization of virtual photons.

\section{Acknowledgments}

T.B is supported by U.S. DOE grant \#DE-FG02-92ER41989. N.H.C and L.C.J are supported by U.S. DOE grant \#de-sc0011941. M.H is supported by Grants-in-Aid for Scientific Research \#25610053. T.I and C.L are supported by U.S. DOE Contract \#AC-02-98CH10996(BNL). T.I is also supported by Grants-in-Aid for Scientific Research \#26400261.

\section{References}

[1] J. Prades, E. de Rafael and A. Vainshtein, Adv. Ser. Direct. High Energy Phys. 20, 303 (2009).

[2] T. Blum, S. Chowdhury, M. Hayakawa and T. Izubuchi, Phys. Rev. Lett. 114, no. 1, 012001 (2015).

[3] T. Blum, N. H. Christ, M. Hayakawa, T. Izubuchi, L. C. Jin and C. Lehner, arXiv:1510.07100 [hep-lat].

[4] J. Green, O. Gryniuk, G. von Hippel, H. B. Meyer and V. Pascalutsa, arXiv:1507.01577 [hep-lat].

[5] T. Blum, M. Hayakawa and T. Izubuchi, PoS LATTICE 2013, 439 (2014).

[6] S. Borsanyi et al., Science 347, 1452 (2015). 